\title{
Case report of anesthesia for free fibula flap reconstruction in Ewing sarcoma: safety and efficacy of Continuous Popliteal Sciatic Nerve Block and very low doses of intravenous Heparin
}

\author{
alessandra di palma ${ }^{1}$, federica grassi ${ }^{2}$, lea pia Cantatore $^{3}$, francesca tortora ${ }^{2}$, francesca \\ grussu $^{2}$, Mario Zama ${ }^{1}$, and SERGIO PICARDO ${ }^{1}$ \\ ${ }^{1}$ Ospedale Pediatrico Bambino Gesù \\ ${ }^{2}$ Ospedale Pediatrico Bambino Gesu \\ ${ }^{3}$ Ospedali Riuniti di Foggia
}

February 14, 2022

\begin{abstract}
A child with a maxillary Ewing sarcoma was operated for tumor asportation and reconstruction with free fibula flap. Adequate anticoagulation was achieved with lower doses of heparin and monitored with multiple ACT values. We used NIRS monitoring to avoid hypoperfusion. Post-operative pain relief was guarantited by local anestethic continous infusion.
\end{abstract}

Case report of anesthesia for free fibula flap reconstruction in Ewing sarcoma: safety and efficacy of Continuous Popliteal Sciatic Nerve Block and very low doses of intravenous Heparin

Di Palma ${ }^{1 *}$, F. Grassi ${ }^{1}$, L. P. Cantatore ${ }^{2}$, F. Tortora ${ }^{1}$, F. Grussu ${ }^{3}$, M. Zama ${ }^{3}$, S.G. Picardo ${ }^{1}$

${ }^{1}$ Anesthesia and Intensive Care Department, Ospedale Pediatrico Bambino Gesù, Roma;

${ }^{2}$ Anesthesia and Intensive Care Department, Ospedali Riuniti di Foggia

${ }^{3}$ Plastic Surgery and Maxillofacial Surgery Department of Ospedale Pediatrico Bambino Gesù, Roma

*Corresponding author: Alessandra Di Palma, ${ }^{1}$ Anesthesia and Intensive Care Department, Ospedale Pediatrico Bambino Gesù Roma.

Email:alessandra.dipalma@opbg.net

ORCID ID: 0000-0003-4302-3811

\section{Abstract}

Keywords

Ewing sarcoma; free fibula flap; continuous popliteal sciatic nerve block; activated clotting time;

\section{Background}

Ewing sarcoma is a malignant lesion of bones, described for the first time in 1921. It occurs predominantly in children and young adults aged between 4 and 25 , has male predilection and affects most commonly long bones but also skull, pelvic girdle, mandible and maxilla.

The diagnosis is difficult and requires clinical, radiological and histopathological correlation. (1) 
This case report describes the anesthesiological management of a case of Ewing sarcoma in a 11-year-old male patient undergoing a hemi-maxillectomy and reconstruction with a free fibula flap.

\section{Case presentation}

A 11-year old Caucasian male, $33 \mathrm{~kg}$, diagnosed with a maxillary Ewing's Sarcoma, was admitted in February 2021 at Plastic Surgery and Maxillofacial Surgery Department of Ospedale Pediatrico Bambino Gesù in Rome for tumor removal and maxillary reconstruction using a free fibula flap.

At arrival in the operating room, standard monitoring with non-invasive arterial blood pressure, 3-lead electrocardiography and oxygen saturation was setted.

After preoxygenation, general anesthesia was induced and naso-tracheal intubation $(6.0 \mathrm{~mm}$ preformed spiral tube) performed. Protective ventilation to maintain normal oxygen and $\mathrm{CO} 2$ end tidal levels in the range 30-40 was provided.

Anesthesia was conducted with Sevoflurane (MAC 1.5-2) and remifentanil (0.2-0.3 mcg/ $/ \mathrm{kg} / \mathrm{min})$ to target BIS values between 40 and 50 . We maintained permissive hypotension during tumor removal and isolation of the fibula flap, and instead higher blood pressure values after vascular anastomosis completion to ensure adequate perfusion of the free flap and its engraftment.

Before the surgical incision, ultrasound-guided Continuous Popliteal Sciatic Nerve Block was performed using a lateral approach.

A linear probe $(8-12 \mathrm{MHz})$ was positioned transversely at the popliteal crease: the popliteal artery and vein were identified at a depth of $3 \mathrm{~cm}$; laterally, biceps femoris muscles and medially the semimembranosus and semitendinosus muscles were identified. At a depth of $5 \mathrm{~cm}$, the tibial and peroneal nerves were visualized joining together to form the sciatic nerve.

Guided by real-time ultrasound, a sterile 22-G Tuohy-type needle was cautiously advanced in-plane. After negative aspiration test, $10 \mathrm{ml}$ of Ropivacaine $0.2 \%$ was administered within the sciatic nerve sheath (Vloka's sheath) to separate both components of the nerve from adipose tissue and muscles. Drug spread was documented proximally and distally to the site of the injection.

The catheter was inserted $5 \mathrm{~cm}$ beyond the needle tip and its correct placement was documented by observing injection of local anesthetic within the sciatic nerve sheath; it was then secured through tunnelling and infusion regimen with Ropivacaine $0.2 \% 5 \mathrm{ml} / \mathrm{h}$ was started.

Surgery started 20 minutes after the block and, based on stable heart rate and blood pressure, there was no need of any rescue analgesia. (2)

As stated before, blood pressure was modulated according to different phases of the surgery. Permissive hypotension was the target during the first part when tumor asportation and preparation of the fibula flap were performed. Differently, during reconstruction phase, especially after completion of the vascular anastomosis and flap reperfusion, constant higher levels of blood pressure were maintained to ensure adequate perfusion of the graft, avoiding hypertensive peaks which instead would increase the risk of damage of the new anastomosis and bleeding.

To decrease the risk of thrombosis at the level of the vascular anastomosis, unfractionated heparin is administered immediately before reperfusion of the graft. Activated clotting time (ACT) was defined as point-of-care method to monitor the adequacy of intraoperative anticoagulation (target ACT >200 seconds).

A baseline ACT value was obtained and, if it was below the threshold on 200 seconds, administration of a bolus of $20 \mathrm{UI} / \mathrm{kg}$ of unfractionated heparin was performed. ACT was repeated 3 minutes later and then at regular intervals until the end of the surgery. Values were always above the threshold so no further administration of heparin was necessary. 
At the end of the surgery a NIRS (Near Infrared Spectroscopy) probe was positioned over the skin area near the vascular anastomosis to monitor oxygenation of the free fibula flap. The probe was kept in place for the first 24 hours after surgery with values always above $92 \%$. The goal was to detect in advance eventual hypoperfusion of the tissues which may contribute to the delay in the engraftment of the flap.

The patient was transferred to the postoperative Intensive Care Unit and was extubated 12 hours after the admission. On the 1st postoperative day the patient was discharged to the maxillofacial surgery ward and few hours later mobilized. The antalgic nerve sciatic catheter has been removed 4 days after surgery. The patient went back home after one week.

\section{Discussion and Conclusions}

Reconstruction of a resected tissue with a free flap involves the transfer of a tissue comprehensive of its vascular peduncle from a donor to a recipient site. In order to ensure adequate blood supply of the free flap and its engraftment in the new body area, reconstruction of the vascular tree performing microvascular anastomosis is necessary. This is especially important in the pediatric population to ensure adequate growth of the graft together with the rest of the body during puberty.

Late engraftment or ischemia with possible successive necrosis of the flap can happen in the postoperative period if constant sufficient blood supply is not provided. Tissue hypoperfusion related to low blood pressure and thrombosis of the new anastomosis are among the most common causes of flap dysfunction.

Concerning hypoperfusion there are different strategies that can be employed, mostly strict monitoring of blood pressure with target normotensive values in the last phases of the surgery (after reperfusion of the graft) and in the first postoperative days, and maintenance of a NIRS probe over the skin area near the vascular anastomosis to detect possible progressive decrease in the NIRS values as sign of increased extraction of oxygen by peripheral tissues and if protracted hypoperfusion.

Thrombosis can occur at the level of venous or arterial anastomosis. Platelet aggregation is the underlying cause of arterial thrombosis whereas venous thrombosis is primarily the result of fibrin clotting and has three main predisposing factors for its development (Virchow's triad): hypercoagulability, stasis and endothelial damage which predisposes to turbulent flow.

There are no evidence-based guidelines for the prevention of microvascular thrombosis after free tissue transfer in head and neck surgery. In practice, most surgical patients receive intravenous heparin $50 \mathrm{UI} / \mathrm{kg}$ in the operating room immediately before the reperfusion of the graft and then, in the postoperative days, prophylaxis of deep vein thrombosis with subcutaneous heparin, often associated with aspirin, milrinone and dextran. The high dosage of heparin administered during the surgery carries a high risk of complications, above all formation of hematomas, thrombocytopenia and bleeding. (3)

In this paper we show that lower UI/kg of unfractionated heparin can be enough to obtain adequate anticoagulation in children and ACT can be a valid method to monitor the response to the initial bolus and, when repeated at regular intervals during the surgery, eventual need of additional heparin. A substantial reduction of the risk of thrombosis is possible avoiding main systemic side effects. The second aspect of this paper is the importance of pain control following free flap microsurgery allowing early mobilization which can be reached with different techniques: parenteral opioids, epidural analgesia and peripheral nerve blocks. The ongoing goal in the fields of anesthesia and surgery is to provide a combination of reliable analgesia while minimizing adverse side effects. In this direction the loco-regional anesthesia is the best option; peripheral nerve blocks carry in fact less side effects compared to epidural anesthesia (epidural hematomas, urinary retention, spinal headache, hypotension, motor weakness, and hemodynamic instability from the sympathectomy) while providing satisfactory pain management both at rest and during mobilization making it well tolerated by the patient. $(3,4)$ In this case, we have seen as a sciatic nerve catheter with continuous infusion of ropivacaine provided effective analgesia for postoperative in patient undergoing free flap microsurgery and how the reduction in pain and side effects allowed for early ambulation, improving postoperative rehabilitation and patient satisfaction, while decreasing length of hospital stay and risk for nosocomial infections. 
Current practices remain extremely diverse and the present report represents an example of avoiding anastomosis thrombosis with very low doses of intravenous heparin and a fast recovery due to perineural continuous block. Further prospective studies could improve the quality of available evidence.

\section{List of abbreviations}

ACT: activated clotting time

NIRS: Near Infrared Spectroscopy

\section{Declarations}

\section{Ethics approval and consent to participate}

Not applicable

\section{Consent for publication}

The patient's parents gave written consent for his personal and clinical details to be published in this study. Availability of data and materials

Data supporting our finding can be found atalessandra.dipalma@opbg.net, the corresponding author.

\section{Competing interests}

Authors declare no competing interests.

\section{Funding}

No funding were received.

\section{Authors'contributions}

ADP conceived and designed the work; FG, LPC, FT and FG have made substantial contributions to the conception of the manuscript; MZ and SGP revised the last version. All authors have read and approved the final manuscript.

\section{Acknowledgements}

Not applicable.

\section{References}

1. Astekar M, Saxena S, Murari A, Manjunatha BS. Ewing's sarcoma in maxilla. BMJ Case Rep. 2019;12(2).

2. Hadzic A, Vloka JD, Hadzic A, Rand F, Khaimova Z, Hadzic N, et al. NYSORA - New York School of Regional Anesthesia - Anesthesia teaching on the web: A tool for the generations to come. Internet J Pain, Symptom Control Palliat Care. 2001;1(2).

3. Askari M, Fisher C, Weniger FG, Bidic S, Lee WPA. Anticoagulation Therapy in Microsurgery: A Review. J Hand Surg Am. 2006;31(5):836-46.

4. Bruegger D, Bauer A, Finsterer U, Bernasconi P, Kreimeier U, Christ F. Microvascular changes during anesthesia: Sevoflurane compared with propofol. Acta Anaesthesiol Scand. 2002;46(5):481-7.

5. Hagau N, Longrois DAN. Anesthesia for free vascularized tissue transfer. Vol. 29, Microsurgery. 2009. p. $161-7$.

6. Motakef S, Mountziaris PM, Ismail IK, Agag RL, Patel A. Emerging paradigms in perioperative management for microsurgical free tissue transfer: Review of the literature and evidence-based guidelines. In: Plastic and Reconstructive Surgery. 2015. p. 290-9. 